\title{
Cramér-Rao Bound Study of Multiple Scattering Effects in Target Localization
}

\author{
Edwin A. Marengo, Maytee Zambrano-Nunez, and Paul Berestesky \\ Department of Electrical and Computer Engineering, Northeastern University, Boston, MA 02115, USA \\ Correspondence should be addressed to Edwin A. Marengo, emarengo@ece.neu.edu
}

Received 4 March 2012; Accepted 1 May 2012

Academic Editor: Francesco Soldovieri

Copyright ( $) 2012$ Edwin A. Marengo et al. This is an open access article distributed under the Creative Commons Attribution License, which permits unrestricted use, distribution, and reproduction in any medium, provided the original work is properly cited.

\begin{abstract}
The target position information contained in scattering data is explored in the context of the scalar Helmholtz operator for the basic two-point scatterer system by means of the statistical estimation framework of the Fisher information and associated CramérRao bound (CRB) relevant to unbiased position estimation. The CRB results are derived for the exact multiple scattering model and, for reference, also for the single scattering or first Born approximation model applicable to weak scatterers. The roles of the sensing configuration and the scattering parameters in target localization are analyzed. Blind spot conditions under which target localization is impossible are derived and discussed for both models. It is shown that the sets of sensing configuration and scattering parameters for which localization is impeded are different but equivalent (they have the same size) under the exact multiple scattering model and the Born approximation. Conditions for multiple scattering to be useful or detrimental to localization are derived.
\end{abstract}

\section{Introduction}

The longstanding question of quantifying the information content about a wave scatterer that is contained in scattered field data has recently been addressed in a number of papers [1-4] via the statistical signal processing framework of the Fisher information and the associated Cramér-Rao bound (CRB) [5]. Motivation for this approach is provided by the practical interest in the role of the physical phenomenon of multiple scattering in either enhancing $[1-4,6]$ or diminishing $[1,4]$ the imaging capabilities relative to the classical reference provided by diffraction theory and, in particular, inverse scattering in the Born approximation. Shi and Nehorai [2] showed via exhaustive numerical computation that multiple scattering can enhance the estimation of scattering parameters of multiple scattering point targets in three-dimensional (3D) space, with particular emphasis on the idea of adding artificial scatterers to enhance estimation of parameters associated to sought-after scatterers. Simonetti et al. [3] showed imaging enhancements from multiple scattering of point targets in 2D space. On the other hand,
Sentenac et al. [1] and Chen and Zhong [4] demonstrated that while multiple scattering can under certain circumstances enhance imaging, it can in other cases be detrimental, in comparison to the baseline provided by the single scattering or first Born approximation signal model. Chen and Zhong [4] provided analytically supported examples of conditions under which the multiple scattering effect is necessarily destructive for electromagnetic transverse-electric (TE) imaging of cylinders. The use of the Fisher information and companion CRB approach is key to conclusively address such fundamental questions since it quantifies the best precision with which scattering parameters can be estimated, in the statistical framework of unbiased estimation under given signal corruption or noise models. This quantification is fundamental. In particular, it is algorithm-independent and showcases the role of both scattering parameters and imaging or sensing configuration. In fact, as is explained by Sentenac et al. [1], and in other related papers [7-10], this is the logical theoretical framework to quantify fundamental imaging limits under multiple scattering where the mapping from object function to data is nonlinear, which prevents 
the direct application of the standard diffraction limits $(\lambda / 2$ rule of thumb) of inverse scattering problems under the Born approximation as well as inverse source problems, where the respective map is linear and therefore tractable via bandlimitation considerations in spatial Fourier domain.

The present paper continues this line of research by investigating, within the scalar Helmholtz wave equation formalism that arises in acoustics, electromagnetics, and optics, the Fisher information and CRB for the fundamental case of two point scatterers in 3D space, with particular emphasis on the problem of target localization. This concrete canonical scattering system is the simplest scatterer exhibiting multiple scattering and provides a mathematically tractable framework to tackle a number of fundamental questions. Particular attention is given to the exact multiple scattering model, but, for reference purposes, we also consider the special case of the first Born approximation applicable to weak scatterers, which sheds insight into the role of multiple scattering on either enhancing or diminishing target localization relative to the baseline provided by the Born approximation. The derived Fisher information and CRB results for two point targets are used to interpret and illustrate through both theoretical analysis and computer examples the roles of the sensing configuration and the scattering parameters on the task of localizing the targets. Concrete conditions are given, backed by the theoretical results, under which localization is facilitated or obstructed, and the two scattering models are comparatively analyzed both analytically and numerically.

The main contributions of the present paper can be summarized as follows. While past focus has centered on the resolution question $[1,3,4]$, we consider the effect of multiple scattering on localization. We derive closedform Fisher information and CRB expressions applicable to the localization of a given two-point target system (whose scattering parameters except its position are known) and exploit the implications of the resulting developments with the aid of computer illustrations. We derive the conditions under which localization is impeded, both under the exact multiple scattering model and in the approximate weak scattering model. It is shown that the sets of sensing configuration and scattering parameters for which localization is impeded are different but equivalent (they have the same size) under the exact multiple scattering model and the Born approximation. We explicitly give the conditions under which multiple scattering enhances or diminishes localizability relative to the reference provided by the first Born approximation. We provide concrete examples where multiple scattering outperforms the Born approximation model with regards to localization (meaning that the Born approximation predictions are unrealistically pessimistic), and vice versa situations where the predictions of the Born approximation model are better (unrealistically optimistic). In addition, the adoption of the canonical system of two point scatterers allows us to gain insight into the role of both sensing configuration and scattering parameters in the localization of targets, and, in particular, the mathematical expressions for the Fisher information and CRB derived in the paper clearly demonstrate factors that depend only on configuration or on scattering parameters as well as more complex factors that depend on both. Furthermore, the formally tractable two targets case is not without many practical applications. The theoretical and computational results of this work relevant to sensing configurations that enhance or diminish information content, including blind spots in the data, and conditions under which multiple scattering is useful or detrimental, have bearings in practical radar and sonar systems interrogating two closely spaced targets, where their own multiple scattering can be used by the targets to reduce their detection or by the system to optimally interrogate them so as to gain maximal information.

\section{Review of the Multiple Scattering Model}

We consider scattering in the context of the Helmholtz operator. Thus, the probing or incident fields $\Psi^{(i)}(\mathbf{r})$ obey

$$
\left(\nabla^{2}+k^{2}\right) \Psi^{(i)}(\mathbf{r})=0,
$$

where $k=2 \pi / \lambda$ is the wavenumber of the field corresponding to wavelength $\lambda$. In the presence of scatterers or inhomogeneities the total field $\Psi(\mathbf{r})$ obeys

$$
\left(\nabla^{2}+k^{2}\right) \Psi(\mathbf{r})=V(\mathbf{r}) \Psi(\mathbf{r})
$$

where $V(\mathbf{r})=k^{2}-\kappa^{2}(\mathbf{r})$, where $\kappa(\mathbf{r})$ denotes the wavenumber of the field in the total medium including the scatterers. From (1) and (2), the scattered field

$$
\Psi^{(s)}(\mathbf{r})=\Psi(\mathbf{r})-\Psi^{(i)}(\mathbf{r})
$$

obeys

$$
\left(\nabla^{2}+k^{2}\right) \Psi^{(s)}(\mathbf{r})=V(\mathbf{r}) \Psi(\mathbf{r}) .
$$

The solution of (4) obeying the radiation condition is given by

$$
\Psi^{(s)}(\mathbf{r})=\int d^{3} r^{\prime} V\left(\mathbf{r}^{\prime}\right) \Psi\left(\mathbf{r}^{\prime}\right) G\left(\mathbf{r}-\mathbf{r}^{\prime}\right),
$$

where Green's function $G$ is given by

$$
G\left(\mathbf{r}-\mathbf{r}^{\prime}\right)=-\frac{e^{i k\left|\mathbf{r}-\mathbf{r}^{\prime}\right|}}{4 \pi\left|\mathbf{r}-\mathbf{r}^{\prime}\right|}
$$

In the far zone where $\left|\mathbf{r}-\mathbf{r}^{\prime}\right| \simeq r-\mathbf{s} \cdot \mathbf{r}^{\prime}$, where $r=|\mathbf{r}|$ and $\mathbf{s}=\mathbf{r} / r$, we have

$$
G\left(\mathbf{r}-\mathbf{r}^{\prime}\right) \sim-\frac{e^{i k r}}{4 \pi r} e^{-i k s \cdot \mathbf{r}^{\prime}},
$$

which upon substitution in (5) yields the expression for the far scattered field:

$$
\Psi^{(s)}(\mathbf{r}) \sim-\frac{e^{i k r}}{4 \pi r} f\left(s ; \Psi^{(i)}\right),
$$

where the quantity $f\left(\mathbf{s} ; \Psi^{(i)}\right)$ is the far-field scattering amplitude and is given by

$$
f\left(\mathbf{s} ; \Psi^{(i)}\right)=\int d^{3} r^{\prime} V\left(\mathbf{r}^{\prime}\right) \Psi\left(\mathbf{r}^{\prime}\right) e^{-i k s \cdot \mathbf{r}^{\prime}} .
$$




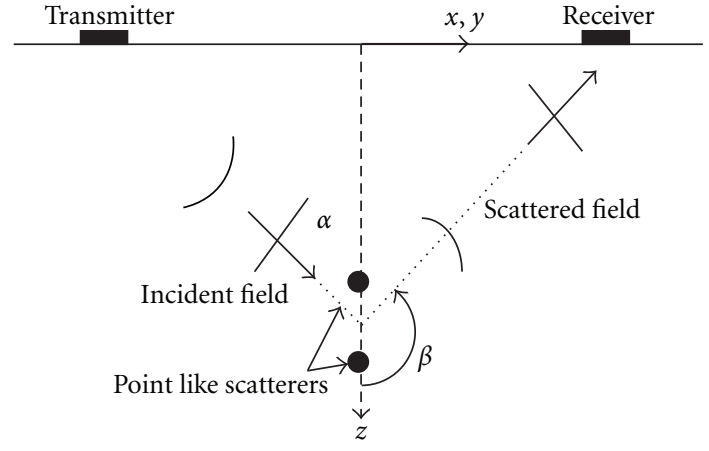

Figure 1: Conceptual illustration of the scattering system.

We focus next on the point scatterer model that is relevant to scatterers having small dimensions compared to the wavelength [11]. This model is well known to be at the heart of many physical models of acoustic, electromagnetic, and quantum scattering (see [12] for an overview of the accuracy and applicability of the point scatterer model in electromagnetics and quantum theory; see also [13] for a relevant distributional interpretation of the point scatterer including a discussion of the associated Foldy-Lax multiple scattering model for point scatterers which is adopted next). In particular, for point targets having locations $\mathbf{R}_{m}, m=$ $1,2, \ldots, M$ and scattering strengths $\tau_{m}, m=1,2, \ldots, M$, we consider the Foldy-Lax multiple scattering model (see, e.g., [13], Tsang et al. [14], page 379), where the scattered field is given by the discrete counterpart of (5):

$$
\Psi^{(s)}(\mathbf{r})=\sum_{m=1}^{M} \tau_{m} \Psi\left(\mathbf{R}_{m}\right) G\left(\mathbf{r}-\mathbf{R}_{m}\right)
$$

where

$$
\Psi\left(\mathbf{R}_{m}\right)=\Psi^{(i)}\left(\mathbf{R}_{m}\right)+\sum_{\substack{n=1 \\ n \neq m}}^{M} \tau_{n} \Psi\left(\mathbf{R}_{n}\right) G\left(\mathbf{R}_{m}-\mathbf{R}_{n}\right) .
$$

By using (7) in the expressions (10) and (11) that define the scattered field, one arrives at (8) where the corresponding scattering amplitude is given by the discrete counterpart of (9):

$$
f\left(\mathbf{s} ; \Psi^{(i)}\right)=\sum_{m=1}^{M} \tau_{m} \Psi\left(\mathbf{R}_{m}\right) e^{-i k \mathbf{s} \cdot \mathbf{R}_{m}}
$$

To fix ideas, let us focus on a system of two point scatterers having complex scattering strengths $\tau_{1}$ and $\tau_{2}$, and positions $\mathbf{R}_{1}=\left(0,0, d_{1}\right)$ and $\mathbf{R}_{2}=\left(0,0, d_{2}\right)$, in the $z$ axis. A conceptual illustration is given in Figure 1. We assume that $d_{2}>d_{1}$. Let the target separation $d=d_{2}-d_{1}>0$. Consider incident plane waves $e^{i k s_{i} \cdot \mathbf{r}}$ with incidence polar angle $\alpha\left(\cos \alpha=\mathbf{s}_{i} \cdot \widehat{\mathbf{z}}\right)$ and far-zone sensing at scattering angle $\beta(\cos \beta=\mathbf{s} \cdot \widehat{\mathbf{z}})$. For this two-scatterer system, and under plane wave excitation, expression (11) yields the system of equations:

$$
\begin{gathered}
\Psi\left(\mathbf{R}_{1}\right)=e^{i k d_{1} \cos \alpha}+\tau_{2} \Psi\left(\mathbf{R}_{2}\right) G(d) \\
\Psi\left(\mathbf{R}_{2}\right)=e^{i k d_{2} \cos \alpha}+\tau_{1} \Psi\left(\mathbf{R}_{1}\right) G(d),
\end{gathered}
$$

where

$$
G(d)=-\frac{e^{i k d}}{4 \pi d},
$$

and whose solution under the nonresonance condition $\tau_{1} \tau_{2} G^{2}(d) \neq 1$, which will be assumed in the following, is given by

$$
\begin{aligned}
& \Psi\left(\mathbf{R}_{1}\right)=F\left(d, \tau_{1}, \tau_{2}\right)\left\{e^{i k d_{1} \cos \alpha}\left[1+\tau_{2} G(d) e^{i k d \cos \alpha}\right]\right\}, \\
& \Psi\left(\mathbf{R}_{2}\right)=F\left(d, \tau_{1}, \tau_{2}\right)\left\{e^{i k d_{1} \cos \alpha}\left[\tau_{1} G(d)+e^{i k d \cos \alpha}\right]\right\},
\end{aligned}
$$

where

$$
F\left(d, \tau_{1}, \tau_{2}\right)=\left[1-\tau_{1} \tau_{2} G^{2}(d)\right]^{-1} .
$$

Note that, for nonzero $\tau_{1}$ and $\tau_{2}, F \rightarrow 0$ as $d \rightarrow 0$ while, for nonzero $d, F \rightarrow 1$ as $\left|\tau_{1} \tau_{2} G^{2}(d)\right| \rightarrow 0$, and generally, for finite parameters $d, \tau_{1}, \tau_{2}, F \neq 0$ and $|F| \neq 1$, facts to be used implicitly in the following.

The scattering amplitude including multiple scattering takes the form:

$$
\begin{aligned}
f(\alpha, \beta)= & F\left(d, \tau_{1}, \tau_{2}\right) e^{i k d_{1} g(\alpha, \beta)} \\
& \times\left[\tau_{1}+\tau_{2} e^{i k d g(\alpha, \beta)}+\tau_{1} \tau_{2} G(d) Q(d, \alpha, \beta)\right],
\end{aligned}
$$

where

$$
g(\alpha, \beta)=\cos \alpha-\cos \beta,
$$

$$
\begin{aligned}
Q(d, \alpha, \beta) & =e^{i k d \cos \alpha}+e^{-i k d \cos \beta} \\
& =2 e^{i k d g(\alpha, \beta) / 2} \cos \left[\frac{k d g^{\prime}(\alpha, \beta)}{2}\right],
\end{aligned}
$$

where we have introduced

$$
g^{\prime}(\alpha, \beta)=\cos \alpha+\cos \beta .
$$

For the special case of weak scatterers, where $\left|\tau_{m} G(d)\right| \ll$ $1, m=1,2$, this takes the first Born approximation form $f(\alpha, \beta) \simeq f_{\text {Born }}(\alpha, \beta)$, where

$$
f_{\text {Born }}(\alpha, \beta)=e^{i k d_{1} g(\alpha, \beta)}\left[\tau_{1}+\tau_{2} e^{i k d g(\alpha, \beta)}\right] .
$$

In these expressions, the angles $\alpha$ and $\beta$ lie in the range $[0, \pi]$.

Note that under the special condition:

$$
Q(d, \alpha, \beta)=0
$$

the exact model in (17) takes, for arbitrary scattering strengths, the quasi-Born approximation form

$$
f(\alpha ; \beta)=F\left(d, \tau_{1}, \tau_{2}\right) e^{i k d_{1} g(\alpha, \beta)}\left[\tau_{1}+\tau_{2} e^{i k d g(\alpha, \beta)}\right] .
$$


This special case will play a part in the target localization analysis.

\section{Fisher Information and Cramér-Rao Bounds of Scattering Parameters}

We consider the signal model:

$$
\widetilde{\mathbf{K}}(\xi)=\mathbf{K}(\xi)+\mathbf{W},
$$

where $\mathbf{K}$ is the noise-free data vector, $\widetilde{\mathbf{K}}$ is the collected noisy data vector, $\xi$ is the estimated parameter vector, and $\mathbf{W}$ is complex Gaussian noise with known variance $\sigma^{2}$. The parameter vector $\xi$ depends on the particular estimation problem under consideration. For example, assuming that the noise variance $\sigma^{2}$ is known, a general problem consists of estimating $d_{1}$ and $d$ and the real and imaginary parts of the scattering strengths, that is,

$$
\xi=\left[d_{1} ; d ; \mathfrak{R}\left\{\tau_{1}\right\} ; \mathfrak{I}\left\{\tau_{1}\right\} ; \mathfrak{R}\left\{\tau_{2}\right\} ; \mathfrak{I}\left\{\tau_{2}\right\}\right],
$$

where $\mathfrak{R}$ and $\mathfrak{I}$ denote the real and imaginary parts, respectively. In this work, we focus on the more specialized case of estimating $d_{1}$ under prior knowledge of the other parameters, in particular, $\xi=d_{1}$, which simulates a canonical target localization problem including internal multiple scattering at the two-target system. This simplification reduces the analytical complexity and gives a lot of mathematical and computational insight into the effects of the sensing configuration and scattering parameters in the estimation of target position.

The data entries $K_{n}, n=1,2, \ldots, N$ of the $N \times 1$ data vector $\mathbf{K}$ are the values of the scattering amplitudes $f\left(\alpha_{n}, \beta_{n}\right)$ measurable in scattering experiments corresponding to given pairs $\left(\alpha_{n}, \beta_{n}\right)$ of incident and scattering angles $\alpha_{n} \in[0, \pi]$ and $\beta_{n} \in[0, \pi]$, respectively. From (17) the entries $K_{n}$ are given by

$$
\begin{aligned}
K_{n}(\xi)= & F\left(d, \tau_{1}, \tau_{2}\right) e^{i k d_{1} g\left(\alpha_{n}, \beta_{n}\right)} \\
& \times\left[\tau_{1}+\tau_{2} e^{i k d g\left(\alpha_{n}, \beta_{n}\right)}+\tau_{1} \tau_{2} G(d) Q\left(d, \alpha_{n}, \beta_{n}\right)\right] .
\end{aligned}
$$

The respective Born approximation is given from (21) by $K_{n}(\xi) \simeq K_{n}^{\text {Born }}(\xi)$, where

$$
K_{n}^{\mathrm{Born}}(\xi)=e^{i k d_{1} g\left(\alpha_{n}, \beta_{n}\right)}\left[\tau_{1}+\tau_{2} e^{i k d g\left(\alpha_{n}, \beta_{n}\right)}\right] .
$$

A fundamental measure of the estimability of the parameters of interest, $\xi$, from the noisy data is the Cramér-Rao lower bound or $\mathrm{CRB}$. The $\mathrm{CRB}, \mathrm{CRB}\left(\xi_{i}\right)$, of the parameter $\xi_{i}$, constitutes a lower bound, achievable under mild conditions, for the variance $\operatorname{var}\left(\hat{\xi}_{i}\right)=E\left[\left(\xi_{i}-\hat{\xi}_{i}\right)^{2}\right]$ (where $E$ denotes the expected value) of any unbiased estimate $\hat{\xi}_{i}$ of the parameter $\xi_{i}$. It is given by the diagonal elements of the so-called Fisher information matrix (FIM) [5, equation (3.20)], in particular,

$$
\operatorname{var}\left(\hat{\xi}_{i}\right) \geq\left[I^{-1}(\xi)\right]_{i, i}=\operatorname{CRB}\left(\xi_{i}\right),
$$

where the $\operatorname{FIM} I(\xi)$ is given by [5, equation (15.52)]

$$
I(\xi)_{i, j}=2 \mathfrak{R}\left[\frac{\partial \mathbf{K}^{H}(\xi)}{\partial \xi_{i}} C_{\widetilde{\mathbf{K}}}^{-1}(\xi) \frac{\partial \mathbf{K}(\xi)}{\partial \xi_{j}}\right],
$$

where $H$ denotes the conjugate transpose, and $C_{\widetilde{\mathbf{K}}}$ is the covariance matrix which in our case is simply $C_{\widetilde{\mathbf{K}}}=\sigma^{2} \mathbf{I}$ where I denotes the $N \times N$ identity matrix. Therefore, (29) reduces to

$$
I(\xi)_{i, j}=2 \sigma^{-2} \mathfrak{R}\left[\frac{\partial \mathbf{K}^{H}(\xi)}{\partial \xi_{i}} \frac{\partial \mathbf{K}(\xi)}{\partial \xi_{j}}\right]
$$

or equivalently to the sum of the FIM of all the observations, in particular,

$$
I(\xi)_{i, j}=\sum_{n=1}^{N} I_{i, j}^{(n)}(\xi)
$$

where the entry $I_{i, j}^{(n)}$ of the FIM $\left(I^{(n)}(\xi)\right)$ of the $n$th scattering experiment, corresponding to incidence angle $\alpha_{n}$ and scattering or sensing angle $\beta_{n}$, is given by

$$
I_{i, j}^{(n)}(\xi)=2 \sigma^{-2} \mathfrak{R}\left[\frac{\partial K_{n}^{*}(\xi)}{\partial \xi_{i}} \frac{\partial K_{n}(\xi)}{\partial \xi_{j}}\right] .
$$

In addition, it is not hard to show that

$$
\operatorname{CRB}\left(\xi_{i}\right) \geq\left[I(\xi)_{i, i}\right]^{-1}
$$

with equality holding if $\xi$ is a scalar, for example, if the target strengths and separation are known, but the position $d_{1}$ is unknown (two-target system localization). Thus, the diagonal entries of the FIM are relevant via (33) as lower bound for the CRB itself, or as the exact CRB when only one scattering parameter is estimated (scalar $\xi$ ). In the following, rather than consider the full FIM matrix (for two or more parameters), which is more difficult to compute analytically, and harder to interpret, we explore closed-form expressions for the Fisher information and CRB relevant to target localization when all the parameters except $d_{1}$ are known (two-target system localization). This task is more analytically tractable and still gives a lot of insight about the information pertinent to localizing targets that is contained in the scattering data.

\section{Target Localization Analysis}

This analysis characterizes the information about the scatterer position, for a known scatterer formed by two point scatterers with given strengths $\tau_{1}$ and $\tau_{2}$ and separation distance $(d)$. From a radar or sonar point of view, the question is up to what point the localizability of the two-point scatterer target is affected by the target parameters $d, \tau_{1}, \tau_{2}$, and the particular remote sensing configuration (in the present case, the angles $\left(\alpha_{n}, \beta_{n}\right)$ for which scattering data are collected).

The localization problem consists in estimating a reference point in the scatterer, for example, $d_{1}$ or the center point $d_{c}=\left(d_{1}+d_{2}\right) / 2$. The Fisher information results using $\xi=d_{1}$ 
and $\xi=d_{c}$ are, of course, the same. In the following, we consider $\xi=d_{1}$. Using $\xi=d_{1}$, the Fisher information $I\left(d_{1}\right)$ is given by (31), where the Fisher information $I^{(n)}\left(d_{1}\right)$ of the $n$th experiment is evaluated by applying (32) to the signal models in (26) and (27). For the Born approximation model based on (27), one obtains

$$
I_{\text {Born }}^{(n)}\left(d_{1}\right)=2 k^{2} g^{2}\left(\alpha_{n}, \beta_{n}\right) \operatorname{SNR}_{\text {Born }}\left(\alpha_{n}, \beta_{n}\right),
$$

where

$$
\operatorname{SNR}_{\text {Born }}(\alpha, \beta)=\frac{\left|\tau_{1}+\tau_{2} e^{i k d g(\alpha, \beta)}\right|^{2}}{\sigma^{2}} .
$$

For the more general multiple scattering model based on (26), one obtains

$$
I^{(n)}\left(d_{1}\right)=2 k^{2} g^{2}\left(\alpha_{n}, \beta_{n}\right) \operatorname{SNR}\left(\alpha_{n}, \beta_{n}\right),
$$

where

$$
\begin{aligned}
& \operatorname{SNR}(\alpha, \beta) \\
& =\sigma^{-2}\left|F\left(d, \tau_{1}, \tau_{2}\right)\right|^{2} \\
& \quad \times\left|\tau_{1}+\tau_{2} e^{i k d g(\alpha, \beta)}+\tau_{1} \tau_{2} G(d) Q(d, \alpha, \beta)\right|^{2} \\
& =\sigma^{-2}\left|F\left(d, \tau_{1}, \tau_{2}\right)\right|^{2} \\
& \quad \times\left\{\sigma^{2} \operatorname{SNR}_{\text {Born }}(\alpha, \beta)\right. \\
& \quad+2 \Re\left[\tau_{1} \tau_{2} G(d) Q(d, \alpha, \beta)\left(\tau_{1}^{*}+\tau_{2}^{*} e^{-i k d g(\alpha, \beta)}\right)\right] \\
& \left.\quad+\frac{2\left|\tau_{1}\right|^{2}\left|\tau_{2}\right|^{2}}{(4 \pi d)^{2}}\left[1+\cos \left(k d g^{\prime}(\alpha, \beta)\right)\right]\right\} .
\end{aligned}
$$

In general, in both the exact multiple scattering and Born approximation contexts, the Fisher information $I^{(n)}\left(d_{1}\right)$ and therefore the associated localizability of the two-target system is seen to depend on the respective SNR and the difference of cosine term $g\left(\alpha_{n}, \beta_{n}\right)$ defined by (18). The latter depends only on the sensing configuration (the incidence and scattering or observation angles). For the sensing configurations where $g\left(\alpha_{n}, \beta_{n}\right)=0$, in particular, the experiments where $\alpha_{n}=\beta_{n}$ which we shall refer to as "line of sight (LOS)" condition, it is not possible to extract information about the two-target system location from the scattering data, this being the case for both weak and strong scattering systems. However, if one captures non-line-ofsight (NLOS) data ( such that $\left.\alpha_{n} \neq \beta_{n}\right)$ then $g\left(\alpha_{n}, \beta_{n}\right) \neq 0$ and one may deduce the sought-after location for nonzero SNR. For a given $\alpha$, the sensing configuration term $\left(g^{2}(\alpha, \beta)\right)$ is maximized if $\beta=\pi$ for $\alpha \in[0, \pi / 2)$, if $\beta=0$ for $\alpha \in$ $(\pi / 2, \pi]$, and if $\beta=0$ or $\pi$ for $\alpha=\pi / 2$. And with these values of $\beta$, the values of $\alpha$ giving the largest $g^{2}(\alpha, \beta)=4$ are $\alpha=0$ (with $\beta=\pi$ ) and $\alpha=\pi$ (with $\beta=0$ ), which correspond to special cases of backscattering-based sensing, where $\beta=\pi-\alpha$ as in monostatic radar and

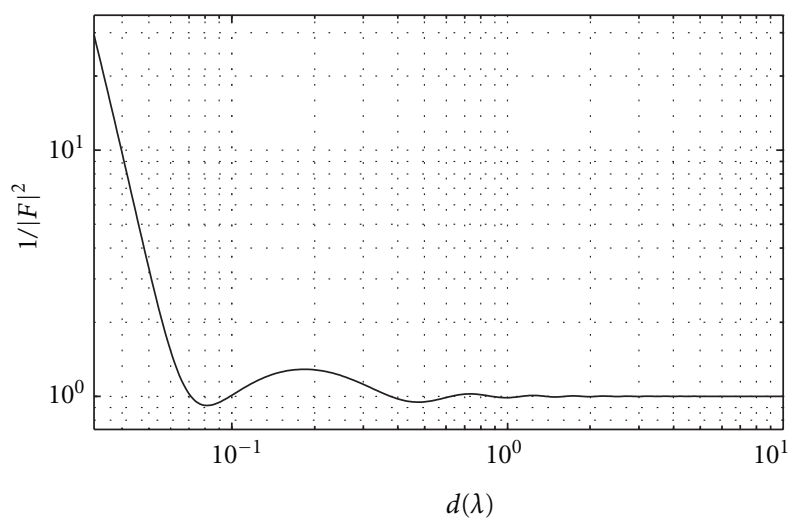

FIgURe 2: Plot of $1 /|F|^{2}$ versus $d$ for $\tau_{1}=1=\tau_{2}$.

sonar systems. In addition, in general the SNR differs in the exact versus approximate models. We see from (37) that in the exact model it depends on the scattering parameters $\left(d, \tau_{1}, \tau_{2}\right)$ through the factor $\left|F\left(d, \tau_{1}, \tau_{2}\right)\right|^{2}$ defined according to (16) and, in a more complex manner, through the large multiplicative parenthesis term in (37), on both the scattering parameters and the sensing configuration. The Born approximation model result (35) does not have the $F$ factor and, in addition, involves only the first of the three terms in the outer parenthesis of the more general result (37). The nature of this important factor is illustrated in Figure 2 which shows a plot of $\left|F\left(d, \tau_{1}, \tau_{2}\right)\right|^{-2}$ (which is proportional to the CRB for the estimation of $\left.d_{1}, \mathrm{CRB}\left(d_{1}\right)\right)$, versus the targets' separation $d$, for fixed values $\tau_{1}=1=\tau_{2}$. To aid interpretation, here and in the subsequent numerical illustrations, we consider the wavenumber $k=2 \pi / \lambda$ for unit-amplitude wavelength $\lambda=1$ so that the value of $d$ shown in the plots is measured directly in wavelengths. Clearly for the case under illustration, the quantity $|F|^{-2}$ is almost unity for moderately large $d(>0.1)$ and grows exponentially as $d \rightarrow 0$. One expects that maximization (minimization) of this scattering-parameter-dependent factor enhances (diminishes) localizability. Furthermore, in the special quasi-Born approximation condition (22) and (23) the exact and approximate models differ only by this factor, so that whenever $\left|F\left(d, \tau_{1}, \tau_{2}\right)\right|>1$ (or $<1$ ), the SNR and Fisher information in the exact model is higher (or lower) than the SNR and information in the approximate model, while if $|F|=1$, the two models perform the same. Then $Q\left(d, \tau_{1}, \tau_{2}\right)=0$ and $|F|>1$ (or $\left.<1\right)$ is a concrete scenario, where the two models can be compared via this factor only.

4.1. Blind Spots for Target Localization. We discuss next concrete examples of "blind spots" for target localization in the space of scattering parameters and sensing angles (values of these parameters and angles for which localization is impeded), as applicable to the exact and approximate models. First we emphasize that no information about the location is contained in LOS data, and this holds for both the exact and approximate models. Another situation when the experiment does not render information is when due 
to the combined role of scattering parameters and sensing configuration the SNR happens to vanish.

Within the Born approximation model, the zero SNR condition is from (35):

$$
\tau_{1}+\tau_{2} e^{i k d g(\alpha, \beta)}=0
$$

There are infinite values of the scattering parameters and the sensing angles $(\alpha, \beta)$ for which this condition is obeyed. In particular, the constraint equation (38) is equivalent to

$$
\begin{aligned}
& \qquad\left|\tau_{1}\right|=\left|\tau_{2}\right|, \\
& k d g(\alpha, \beta) \\
& =k d(\cos \alpha-\cos \beta) \\
& =\theta_{1}-\theta_{2}+l \pi, \quad l= \pm(2 p+1), p=0,1,2, \ldots, p_{\max },
\end{aligned}
$$

where the real-valued $\theta_{1}, \theta_{2}$ are the phase angles of $\tau_{1}, \tau_{2}$, that is, $\tau_{1}=\left|\tau_{1}\right| e^{i \theta_{1}}, \tau_{2}=\left|\tau_{2}\right| e^{i \theta_{2}}$. Since the maximum value of $|g(\alpha, \beta)|=2$, then $p_{\max }$ is the maximum value of integer index $p \geq 0$ in (39) that obeys $\left|\left(\theta_{1}-\theta_{2}\right) / \pi \pm(2 p+1)\right| \leq 4 d / \lambda$, that is,

$$
(2 p+1)^{2} \pm \frac{2(2 p+1)\left(\theta_{1}-\theta_{2}\right)}{\pi}+\frac{\left(\theta_{1}-\theta_{2}\right)^{2}}{\pi^{2}} \leq 16\left(\frac{d}{\lambda}\right)^{2} .
$$

For example, if $\tau_{1}=\tau_{2}$ then from (39) if the condition $k d g(\alpha, \beta)=l \pi, l= \pm(2 p+1), p=0,1,2, \ldots, p_{\max }$ holds, then $\operatorname{SNR}_{\text {Born }}(\alpha, \beta)=0$. Given any choice of the angles $\alpha, \beta$, the target separations $d=(2 p+1) \lambda /[2|g(\alpha, \beta)|]>0, p=$ $0,1,2, \ldots$ render zero $\mathrm{SNR}, \operatorname{SNR}_{\mathrm{Born}}(\alpha, \beta)=0$. Alternatively, for any separation distance $d$, then $\operatorname{SNR}_{\text {Born }}(\alpha, \beta)=0$ for the angles $\alpha, \beta$ such that $g= \pm(2 p+1) \lambda / 2 d, p=0,1,2, \ldots, p_{\max }$, where $p_{\max }$ is defined according to the discussion in (40) with $\theta_{1}=\theta_{2}$. In passing, we note that the smallest $d$ for which condition (40) with $\theta_{1}=\theta_{2}$ can be obeyed is $d=\lambda / 4$ (giving $p_{\text {max }}=0$ ), hence for $d<\lambda / 4, \mathrm{SNR}_{\text {Born }} \neq 0$ for the same scatterer strength case, $\tau_{1}=\tau_{2}$, as is illustrated in one of the plots in the numerical illustration section (see Figure 6(b)).

Thus clearly under the Born approximation model, there are values of the parameters and the sensing angles which can be thought of as "blind spots" for target data acquisition, that is, they correspond to zero SNR signals and information. Referring to the more general multiple scattering model result (37), we see that the vanishing of the SNR under the Born approximation does not imply the corresponding vanishing of SNR under the more general multiple scattering model. On the other hand, blind spots are also possible under multiple scattering. To illustrate this with a concrete example, we note that if $\operatorname{SNR}_{\mathrm{Born}}(\alpha, \beta)=0$ so that (39) and (40) are obeyed, then it follows from (37) that $\operatorname{SNR}(\alpha, \beta)=0$ if and only if $Q(d, \alpha, \beta)=0$, which is the quasi-Born approximation condition discussed in (22) and (23). According to (19), this restriction can be stated as

$$
\begin{aligned}
& k d g^{\prime}(\alpha, \beta) \\
& \quad=k d(\cos \alpha+\cos \beta) \\
& \quad=l \pi, \quad l= \pm(2 p+1), \quad p=0,1,2, \ldots .
\end{aligned}
$$

Thus if conditions (39), (40), and (41) are all obeyed under the constraints $|\cos \alpha| \leq 1$ and $|\cos \beta| \leq 1$, then $\operatorname{SNR}(\alpha, \beta)=0$. The general condition can be stated as

$$
\begin{gathered}
\cos \alpha=\frac{\lambda}{4 d}\left[l_{1}+l_{2}+\frac{\left(\theta_{2}-\theta_{1}\right)}{\pi}\right], \\
\cos \beta=\frac{\lambda}{4 d}\left[l_{2}-l_{1}-\frac{\left(\theta_{2}-\theta_{1}\right)}{\pi}\right], \\
l_{1}= \pm\left(2 p_{1}+1\right), \quad p_{1}=0,1,2, \ldots, \\
l_{2}= \pm\left(2 p_{2}+1\right), \quad p_{2}=0,1,2, \ldots, \\
|\cos \alpha|=\left|\frac{\lambda}{d}\left[\frac{l_{1}+l_{2}}{4}+\frac{\left(\theta_{2}-\theta_{1}\right)}{2 \pi}\right]\right| \leq 1, \\
|\cos \beta|=\left|\frac{\lambda}{d}\left[\frac{l_{2}-l_{1}}{4}+\frac{\left(\theta_{2}-\theta_{1}\right)}{2 \pi}\right]\right| \leq 1,
\end{gathered}
$$

where asabove mentioned the real-valued $\theta_{1}, \theta_{2}$ are the phase angles of $\tau_{1}, \tau_{2}$, that is, $\tau_{1}=\left|\tau_{1}\right| e^{i \theta_{1}}, \tau_{2}=\left|\tau_{2}\right| e^{i \theta_{2}}$. An example is the case $\tau_{1}=\tau_{2}$, and $l_{1}=l=l_{2}$, which implies $\beta=\pi / 2$, which further implies $d=l \lambda /(2 \cos \alpha)>0, l= \pm(2 p+1)$, $p=0,1,2, \ldots$.

Another important issue is the implications of these results for the case of monostatic radar or sonar observations, where for a given incidence angle $\alpha$ one captures data for the backscattering direction associated to $\beta=\pi-\alpha$. In this special case, condition (41) cannot be obeyed; therefore, the second and third terms in (37) do not generally vanish. On the other hand, the first term in (37) vanishes if condition (39) holds, where in this case $\cos \beta=-\cos \alpha$, and this can in fact happen. For example, if $\tau_{1}=\tau_{2}$, and $d=\lambda / 4$, then for $\alpha=0$ (or for $\alpha=\pi$ ) the backscattering $\operatorname{SNR}_{\text {Born }}(\alpha=$ $0, \pi-\alpha=\pi)=0$ (or $\operatorname{SNR}_{\text {Born }}(\alpha=\pi, \pi-\alpha=0)=$ $0)$. In this case, however, the exact $\operatorname{SNR}(\alpha=0, \pi-\alpha) \neq 0$ due to the nonvanishing of the second and third terms in (37) as we discuss in the numerical illustrations section. This is a concrete example of a situation where multiple scattering enhances the sensing capabilities, in this case, the localizability, relative to what one would have expected from the approximate model.

In the following, we expand the analysis of the nonlocalizability conditions. We establish the fundamental result that the nonlocalizable sets of sensing configuration and scattering parameters of the two models are actually of the same size.

4.2. Nonlocalizability Conditions. We elaborate the necessary and sufficient conditions for impossible target localizability (nonlocalizability conditions or blind spots). Targets can be located if and only if such nonlocalizability conditions are not obeyed. The derived conditions will be later compared with those for nonlocalizability under the Born approximation, and it will be conclusively demonstrated that the set of values of $\left(d, \tau_{1}, \tau_{2}, \alpha_{n}, \beta_{n}\right)$ yielding nonlocalizability under the Born approximation is of the same size as the set of values of $\left(d, \tau_{1}, \tau_{2}, \alpha_{n}, \beta_{n}\right)$ yielding nonlocalizability under the exact scattering model. This is very important because it conclusively establishes at least for the basic two-point 
target system that it is not true that multiple scattering is usually beneficial in enhancing localization and imaging, but that on the contrary the two models are comparable at least regarding the sizes of their localizable and nonlocalizable sets of values of the combined scattering and configuration parameters.

As discussed earlier, localizability is impeded for identical angles $\alpha_{n}=\beta_{n}$. This is the trivial LOS condition discussed at the beginning of this section. Nonlocalizability can also occur for the nontrivial case $\alpha_{n} \neq \beta_{n}$ if and only if the following condition holds. Since $F \neq 0$ for finite values of $d, \tau_{1}, \tau_{2}$, then under the exact scattering model (36) and (37) $I^{(n)}\left(d_{1}\right)=0$ for $\alpha_{n} \neq \beta_{n}$ if and only if

$$
\tau_{1}+\tau_{2} e^{i k d g\left(\alpha_{n}, \beta_{n}\right)}+\tau_{1} \tau_{2} G(d) Q\left(d, \alpha_{n}, \beta_{n}\right)=0 .
$$

There are infinite values of the scattering and configuration parameters for which this condition is obeyed. For instance, one may choose any allowed value of the angles $\alpha_{n} \in[0, \pi]$ and $\beta_{n} \in[0, \pi]$ and of the targets' separation $d>0$, as well as any $\tau_{1} \in \mathbb{C}$, which makes (43) a linear equation with one unknown $\left(\tau_{2}\right)$, so that there is a unique value of $\tau_{2}$ obeying this condition. Therefore, the dimensionality of the nonlocalizable set of scattering and configuration parameters $\left(d, \tau_{1}, \tau_{2}, \alpha_{n}, \beta_{n}\right)$ is $5\left(d, \alpha_{n}\right.$, and $\beta_{n}$ are real, while $\tau_{1}$ and $\tau_{2}$ are complex) which represents a significant dimensionality reduction of the entire parameter space which is 7 . Thus, if the scattering and configuration parameters are randomly selected, with overwhelming probability, target localizability is not impeded. Only under the restricted nonlocalizability condition (43) which applies to a reduced subset of parameter space, the two-target system cannot be located.

The respective Born approximation analysis is as follows. As explained in the discussion in Section 4.1 regarding (38), the necessary and sufficient condition for nonlocalizability in the nontrivial case $\alpha_{n} \neq \beta_{n}$ is (38) with $\alpha=\alpha_{n}$ and $\beta=\beta_{n}$. As in the preceding exact multiple scattering result, one can without loss of generality fix the values of $d, \alpha_{n}$, and $\beta_{n}$, as well as assign to $\tau_{1}$ any complex value, and subsequently compute via (38) the unique value $\tau_{2}$ which obeys this relation. As in the multiple scattering discussion, this means the dimensionality of the nonlocalizable parameter set is 5, while the entire parameter set has dimensionality 7 . Furthermore, for any nonlocalizable state built above in the exact scattering framework for fixed $d, \alpha_{n}, \beta_{n}$ and any one of the scattering strengths, one can always build a counterpart and unique nonlocalizable state for the approximate model, and vice versa, so the two sets are linked in a oneto-one manner (they are mathematically equivalent sets), which demonstrates that when studied in the wholeness of parameter space, the exact multiple scattering case cannot be regarded as informationally richer than the approximate model. In addition, we recall that we showed earlier in the discussion in Section 4.1 regarding (41) that the necessary and sufficient condition for $I^{(n)}\left(d_{1}\right)=0, I_{\mathrm{Born}}^{(n)}\left(d_{1}\right)=0$ is (38) and $Q=0$. A summary of the derived relations between the two nonlocalizable sets for the exact and approximate models is shown in Figure 3. Note that based on the discussion above the two sets are of the same size. We have completed

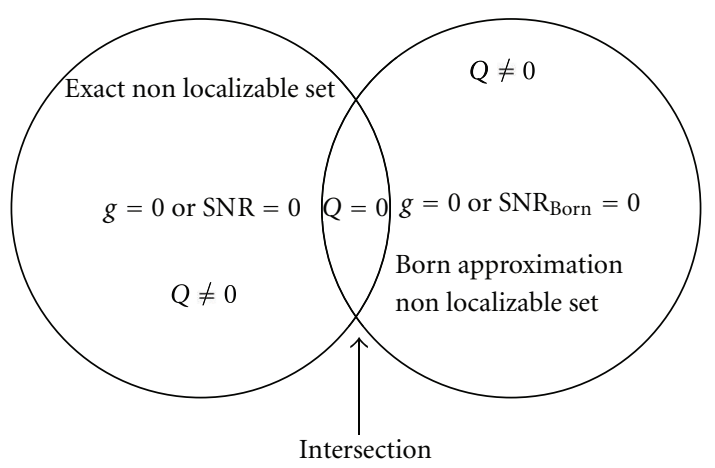

FIGURE 3: Venn diagram illustrating the relations between the nonlocalizable sets in configuration and parameter space for the exact and approximate models. Note that, as shown in the paper, the two sets are of the same size, and the intersection between them is defined by the condition $Q=0$.

the picture by discussing the intersection condition $Q=0$ illustrated in the figure.

4.3. Analog Comparative Analysis. We have shown that in the entire parameter space the two models are comparable with regards to localizability. However, this particular point of view is binary (information is zero or nonzero), so it does not apply to the actual numerical information values which may tend to be higher in either model. To complete the analytical picture, we derive necessary and sufficient conditions for the target localizability, as measured by the Fisher information and associated CRB, to be greater in either scattering model. The following results can be thought of as the analog complement of the binary nonlocalizability (versus localizability) results derived above.

We derive next conditions, for $I^{(n)}\left(d_{1}\right)>I_{\text {Born }}^{(n)}\left(d_{1}\right)$ and for the opposite $I^{(n)}\left(d_{1}\right)<I_{\text {Born }}^{(n)}\left(d_{1}\right)$. First of all, this is possible only if $g \neq 0$ (which we assume next) since for $g=0$ both information vanish as we have elaborated earlier.

Necessary and Sufficient Condition. $I^{(n)}\left(d_{1}\right)>I_{\text {Born }}^{(n)}\left(d_{1}\right)$ : It follows from (34), (35), (36), and (37) that $I^{(n)}\left(d_{1}\right)>$ $I_{\text {Born }}^{(n)}\left(d_{1}\right)$ if and only if $g \neq 0$ and SNR $>\mathrm{SNR}_{\text {Born }}$, in particular,

$$
\begin{gathered}
\left|F\left(d, \tau_{1}, \tau_{2}\right)\right|\left|\tau_{1}+\tau_{2} e^{i k d g\left(\alpha_{n}, \beta_{n}\right)}+\tau_{1} \tau_{2} G(d) Q\left(d, \alpha_{n}, \beta_{n}\right)\right| \\
>\left|\tau_{1}+\tau_{2} e^{i k d g\left(\alpha_{n}, \beta_{n}\right)}\right| .
\end{gathered}
$$

As a special case, this implies that if $Q\left(d, \alpha_{n}, \beta_{n}\right)=0$, then the necessary and sufficient condition for $I^{(n)}\left(d_{1}\right)>I_{\text {Born }}^{(n)}\left(d_{1}\right)$ is

$$
\left|F\left(d, \alpha_{n}, \beta_{n}\right)\right|>1
$$


Sufficient Conditions A. $I^{(n)}\left(d_{1}\right)>I_{\text {Born }}^{(n)}\left(d_{1}\right)$ : Furthermore, the result (44) also implies, via the reverse triangle inequality, the following sufficient condition for $I^{(n)}\left(d_{1}\right)>I_{\text {Born }}^{(n)}\left(d_{1}\right)$ :

$$
\begin{aligned}
& \left|\tau_{1} \tau_{2} G(d) Q\left(d, \alpha_{n}, \beta_{n}\right)\right| \\
& \quad>\left[\left|F^{-1}\left(d, \tau_{1}, \tau_{2}\right)\right|+1\right]\left|\tau_{1}+\tau_{2} e^{i k d g\left(\alpha_{n}, \beta_{n}\right)}\right|,
\end{aligned}
$$

as well as the following sufficient condition for $I^{(n)}\left(d_{1}\right)>$ $I_{\text {Born }}^{(n)}\left(d_{1}\right)$,

$$
\begin{gathered}
\left|F\left(d, \tau_{1}, \tau_{2}\right)\right|>1, \\
\left|\tau_{1} \tau_{2} G(d) Q\left(d, \alpha_{n}, \beta_{n}\right)\right| \\
<\left[1-\left|F^{-1}\left(d, \tau_{1}, \tau_{2}\right)\right|\right]\left|\tau_{1}+\tau_{2} e^{i k d g\left(\alpha_{n}, \beta_{n}\right)}\right| .
\end{gathered}
$$

Necessary Condition $A^{\prime} . I^{(n)}\left(d_{1}\right)>I_{\text {Born }}^{(n)}\left(d_{1}\right)$ : It also follows from (44) and the triangle inequality that a necessary condition for (44) to hold is

$$
\begin{aligned}
& \left|\tau_{1} \tau_{2} G(d) Q\left(d, \alpha_{n}, \beta_{n}\right)\right| \\
& \quad>\left[\left|F^{-1}\left(d, \tau_{1}, \tau_{2}\right)\right|-1\right]\left|\tau_{1}+\tau_{2} e^{i k d g\left(\alpha_{n}, \beta_{n}\right)}\right| .
\end{aligned}
$$

Sufficient Condition $A^{\prime \prime} . I_{\mathrm{Born}}^{(n)}\left(d_{1}\right) \geq I^{(n)}\left(d_{1}\right)$ : A corollary of the necessary condition $A^{\prime}$ in (48) is that the following is a sufficient condition for $I_{\mathrm{Born}}^{(n)}\left(d_{1}\right) \geq I^{(n)}\left(d_{1}\right)$ :

$$
\begin{gathered}
\left|F\left(d, \tau_{1}, \tau_{2}\right)\right|<1, \\
\left|\tau_{1} \tau_{2} G(d) Q\left(d, \alpha_{n}, \beta_{n}\right)\right| \\
\leq\left[\left|F^{-1}\left(d, \tau_{1}, \tau_{2}\right)\right|-1\right]\left|\tau_{1}+\tau_{2} e^{i k d g\left(\alpha_{n}, \beta_{n}\right)}\right| .
\end{gathered}
$$

This condition is related to and complements sufficient condition $A$ discussed above. It can be shown that this result is actually stronger, using $<$ in place of $\leq$ in the second equation it is a sufficient condition for $I_{\text {Born }}^{(n)}\left(d_{1}\right)>I^{(n)}\left(d_{1}\right)$ (strict inequality).

Like its counterpart (44), the necessary and sufficient condition for $I_{\text {Born }}^{(n)}\left(d_{1}\right)>I^{(n)}\left(d_{1}\right)$ is $g\left(\alpha_{n}, \beta_{n}\right) \neq 0$ and

$$
\begin{aligned}
& \left|F\left(d, \tau_{1}, \tau_{2}\right)\right|\left|\tau_{1}+\tau_{2} e^{i k d g\left(\alpha_{n}, \beta_{n}\right)}+\tau_{1} \tau_{2} G(d) Q\left(d, \alpha_{n}, \beta_{n}\right)\right| \\
& <\left|\tau_{1}+\tau_{2} e^{i k d g\left(\alpha_{n}, \beta_{n}\right)}\right| .
\end{aligned}
$$

On the other hand, if $Q\left(d, \alpha_{n}, \beta_{n}\right)=0$, then the necessary and sufficient condition for $I_{\mathrm{Born}}^{(n)}\left(d_{1}\right)>I^{(n)}\left(d_{1}\right)$ is

$$
\left|F\left(d, \tau_{1}, \tau_{2}\right)\right|<1 .
$$

Sufficient Conditions B. $I_{\text {Born }}^{(n)}\left(d_{1}\right)>I^{(n)}\left(d_{1}\right)$ : Moreover, by means of an analysis based on the reverse triangle inequality which is similar to the one leading to the sufficient conditions
$A$ one can also show that a sufficient condition for $I_{\text {Born }}^{(n)}\left(d_{1}\right)>$ $I^{(n)}\left(d_{1}\right)$ is

$$
\begin{aligned}
& \mid \tau_{1} \tau_{2} G(d) Q\left(d, \alpha_{n}, \beta_{n}\right) \mid \\
&> {\left[\left|F\left(d, \tau_{1}, \tau_{2}\right)\right|+1\right] } \\
& \quad \times\left|\tau_{1}+\tau_{2} e^{i k d g\left(\alpha_{n}, \beta_{n}\right)}+\tau_{1} \tau_{2} G(d) Q\left(d, \alpha_{n}, \beta_{n}\right)\right| .
\end{aligned}
$$

Another sufficient condition for $I_{\mathrm{Born}}^{(n)}\left(d_{1}\right)>I^{(n)}\left(d_{1}\right)$ arising from the same analysis is

$$
\begin{gathered}
\left|F\left(d, \tau_{1}, \tau_{2}\right)\right|<1, \\
\left|\tau_{1} \tau_{2} G(d) Q\left(d, \alpha_{n}, \beta_{n}\right)\right| \\
<\left[1-\left|F\left(d, \tau_{1}, \tau_{2}\right)\right|\right] \\
\times\left|\tau_{1}+\tau_{2} e^{i k d g\left(\alpha_{n}, \beta_{n}\right)}+\tau_{1} \tau_{2} G(d) Q\left(d, \alpha_{n}, \beta_{n}\right)\right| .
\end{gathered}
$$

Necessary Condition $B^{\prime} . I_{\mathrm{Born}}^{(n)}\left(d_{1}\right)>I^{(n)}\left(d_{1}\right)$ : Also, it follows from $(50)$ that a necessary condition for $I_{\mathrm{Born}}^{(n)}\left(d_{1}\right)>I^{(n)}\left(d_{1}\right)$ is

$$
\begin{aligned}
& \mid \tau_{1} \tau_{2} G((d) Q\left(d, \alpha_{n}, \beta_{n}\right) \mid \\
&> {\left[\left|F\left(d, \tau_{1}, \tau_{2}\right)\right|-1\right] } \\
& \quad \times\left|\tau_{1}+\tau_{2} e^{i k d g\left(\alpha_{n}, \beta_{n}\right)}+\tau_{1} \tau_{2} G(d) Q\left(d, \alpha_{n}, \beta_{n}\right)\right|
\end{aligned}
$$

Sufficient Condition $B^{\prime \prime} . I^{(n)}\left(d_{1}\right) \geq I_{\text {Born }}^{(n)}\left(d_{1}\right)$ : A corollary of necessary condition $B^{\prime}$ is the following sufficient condition for $I^{(n)}\left(d_{1}\right) \geq I_{\text {Born }}^{(n)}\left(d_{1}\right)$ :

$$
\begin{gathered}
\left|F\left(d, \tau_{1}, \tau_{2}\right)\right|>1, \\
\left|\tau_{1} \tau_{2} G(d) Q\left(d, \alpha_{n}, \beta_{n}\right)\right| \\
\leq\left[\left|F\left(d, \tau_{1}, \tau_{2}\right)\right|-1\right] \\
\times\left|\tau_{1}+\tau_{2} e^{i k d g\left(\alpha_{n}, \beta_{n}\right)}+\tau_{1} \tau_{2} G(d) Q\left(d, \alpha_{n}, \beta_{n}\right)\right|,
\end{gathered}
$$

which is related to and complements sufficient conditions $B$ established above. This result is actually stronger, using the strict inequality in the second equation it is a sufficient condition for $I^{(n)}\left(d_{1}\right)>I_{\text {Born }}^{(n)}\left(d_{1}\right)$, as can be shown independently from (44).

The above conditions are rather straightforward yet very useful expressions that allow one to know a priori without doing the Fisher information and CRB calculations if under given conditions of interest the multiple scattering is beneficial or detrimental to localization relative to the Born approximation predictions. Consider, for example, the first of the conditions $A$. Letting $\tau_{1}=\tau=\tau_{2}$, we find from (46) that if

$$
|\tau G(d)|>\left[\left|F^{-1}(d, \tau, \tau)\right|+1\right]\left|\frac{\cos \left[k d g\left(\alpha_{n}, \beta_{n}\right) / 2\right]}{\cos \left[k d g^{\prime}\left(\alpha_{n}, \beta_{n}\right) / 2\right]}\right|,
$$


then we know without the need of further calculations that the multiple scattering events facilitate greater localization information than the first-order or single-scattering signal alone, and superresolution beyond the Born approximation limits is accessible. As a special case, consider the vanishing of $\cos \left[k d g\left(\alpha_{n}, \beta_{n}\right) / 2\right]$, which corresponds to $\mathrm{SNR}_{\mathrm{Born}}=0$. As long as the denominator in (56) does not vanish, the exact model information is necessarily greater than zero since $|\tau G(d)|>0$, in agreement with the results derived earlier in the paper, since the only way the denominator vanishes is if $Q\left(d, \alpha_{n}, \beta_{n}\right)=0$ (which makes the condition above useless due to the resulting $0 / 0$ indeterminacy) which is in essence the result summarized in the Venn diagram in Figure 3. In the special case of backscattering data, the sufficient condition (56) becomes

$$
|\tau G(d)|>\left[\left|F^{-1}(d, \tau, \tau)\right|+1\right]\left|\cos \left(k d \cos \alpha_{n}\right)\right| .
$$

One of the implications is that if $\cos \left(k d \cos \alpha_{n}\right)=0$, that is, $k d \cos \alpha_{n}=l \pi / 2, l= \pm(2 p+1), p=0,1,2, \ldots$, then the exact multiple-scattering always enhances the backscattering localization information relative to the single-scattering data. This is consistent with the discussion on backscattering at the end of Section 4.1.

\section{Numerical Illustrations}

5.1. Single Observation. Next we discuss a selection of single observation or single-input single-output (SISO) experiments, which illustrate how variations in the system's parameters (scatterers' separation $d$, scatterer strengths $\left(\tau_{1}, \tau_{2}\right)$ ) and observer configuration (incident and observation angles $\alpha_{n}$ and $\beta_{n}$ ) affect the estimation of scatterer location. In generating the following plots, we use $\sigma^{2}=1$ so that the plotted CRB results are normalized by the noise variance $\sigma^{2}$. We consider unit value wavelength $\lambda=1$ so that the wavenumber $k=2 \pi / \lambda=2 \pi$ and all distances (e.g., $d$ ) can be given in the plots in terms of the wavelength.

First we examine the effect on localization information, as described by $\operatorname{CRB}\left(d_{1}\right)$, of the direction of the receiver, $\beta_{1}=\beta$, for the SISO experiment corresponding to incidence angle $\alpha_{1}=\alpha=0$. Figure 4 shows two plots of $\operatorname{CRB}\left(d_{1}\right)$ versus $\beta$ for $\alpha=0$ and $\tau_{1}=1=\tau_{2}$. The two plots shown correspond to $d=\lambda / 4$ and $d=\lambda / 2$, respectively. The general tendency is for $\operatorname{CRB}\left(d_{1}\right)$ to be larger for the small angles $\beta$ near zero and to be smaller for the large angles $\beta$ near $\pi$. Thus it seems that as a rule of thumb one should expect to extract more location information from backscattering and nearbackscattering data than from forward scattering and nearforward scattering experiments. This is anticipated from the discussion following (37), since the Fisher information is proportional to $g^{2}$ which for $\alpha=0$ is maximal at $\beta=\pi$. However, we also expect from the discussion in (42) that for $d=\lambda / 2, \operatorname{CRB}\left(d_{1}\right)=\infty$ for $\beta=\pi / 2$, and this is, in fact, the behavior shown in the figure. Thus for $d=\lambda / 2$, $\operatorname{CRB}\left(d_{1}\right)$ decays with $\beta$ except in the approximate interval $(\pi / 3, \pi / 2)$ where it grows as it reaches a peak at $\beta=\pi / 2$, and then decays steadily beyond $\pi / 2$ up to $\pi$. In contrast, for $d=\lambda / 4, \operatorname{CRB}\left(d_{1}\right)$ decays with $\beta$ in the approximate interval

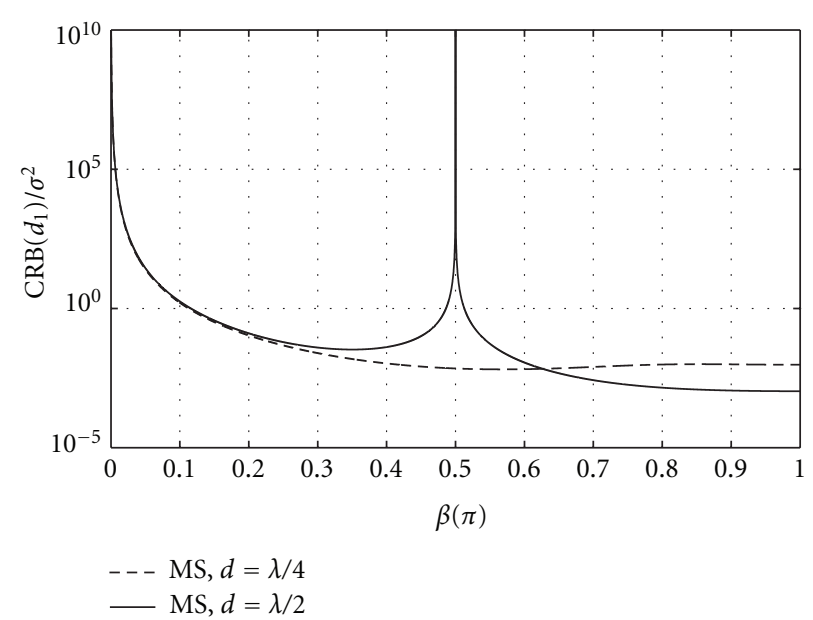

FIGURE 4: $\mathrm{CRB}\left(d_{1}\right)$ corresponding to scattering strength $\tau_{1}=1=\tau_{2}$ and incidence angle $\alpha=0$, as a function of the observation angle $\beta$, for scatterers' separations $d=\lambda / 4$ and $d=\lambda / 2$.

$(0,0.6 \pi)$ and levels off after rising slightly for $\beta \gtrsim 0.6 \pi$ up to $\pi$. It is important to note that, as explained in the paragraph following the discussion of (42), under the Born approximation the location information vanishes completely for $d=\lambda / 4$ if $\beta=\pi$. In contrast, as shown in the plot which corresponds to the multiple scattering model, for these values ( $d=\lambda / 4$ and $\beta=\pi$ ) in general the Fisher information does not vanish (and the respective CRB is not infinity) under the multiple scattering model. This illustrates a (backscattering) scenario where multiple scattering enables information that is not present in the Born approximation model or for weak targets.

Figure 5 shows plots of $\operatorname{CRB}\left(d_{1}\right)$ versus $\alpha_{1}=\alpha$ for backscattering experiments for which the observation angle $\beta_{1}=\beta=\pi-\alpha$. In these calculations, $\tau_{1}=1=\tau_{2}$. Overlaid plots for the multiple scattering and Born approximation models are shown for $d=\lambda / 4$ and $d=\lambda / 2$. For $\alpha=\pi / 2$ and the corresponding $\beta=\pi / 2, g=0$ so that from (34) and $(36) \operatorname{CRB}\left(d_{1}\right)=\infty$ for any values of the scattering parameters ( $d$ included), as shown in these plots. For $d=$ $\lambda / 4$, the Born approximation model requires in view of (39) and (40) that $\operatorname{CRB}\left(d_{1}\right)=\infty$ when $\alpha=0, \pi$, and this is the behavior shown in the respective plot (Figure 5(a)). This example was discussed at the end of Section 4.1, where we also explained why the multiple scattering model does not exhibit these peaks. The exact CRB is almost the same as the approximate $\mathrm{CRB}$ for a broad angular range $(\pi / 4 \lesssim$ $\alpha \lesssim 3 \pi / 4)$. However, it is significantly lower than the Born approximation value elsewhere, particularly near the forward scattering and backscattering angles (angles close to $\alpha=0$ and $\pi$, resp.), where the Born approximation bound peaks occur. For $d=\lambda / 2$, the Born approximation model requires in view of (39) and (40) that $\operatorname{CRB}\left(d_{1}\right)=\infty$ when $\alpha=\pi / 3,2 \pi / 3$, and this is the behavior illustrated in Figure 5(b). Here the situation is different (than in Figure $5(\mathrm{a}))$ in that although the multiple scattering model does not have peaks at $\alpha=\pi / 3,2 \pi / 3$, it does have peaks 


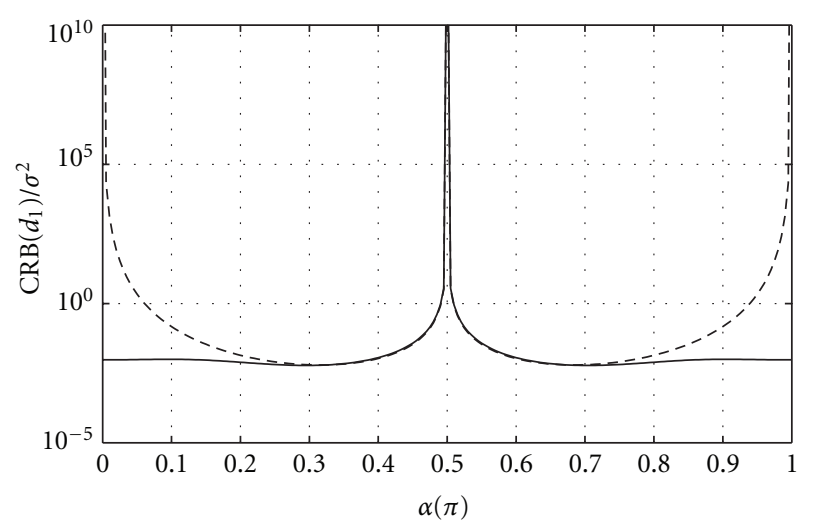

- MS, $d=\lambda / 4$

--- Born $=\lambda / 4$

(a) $d=\lambda / 4$

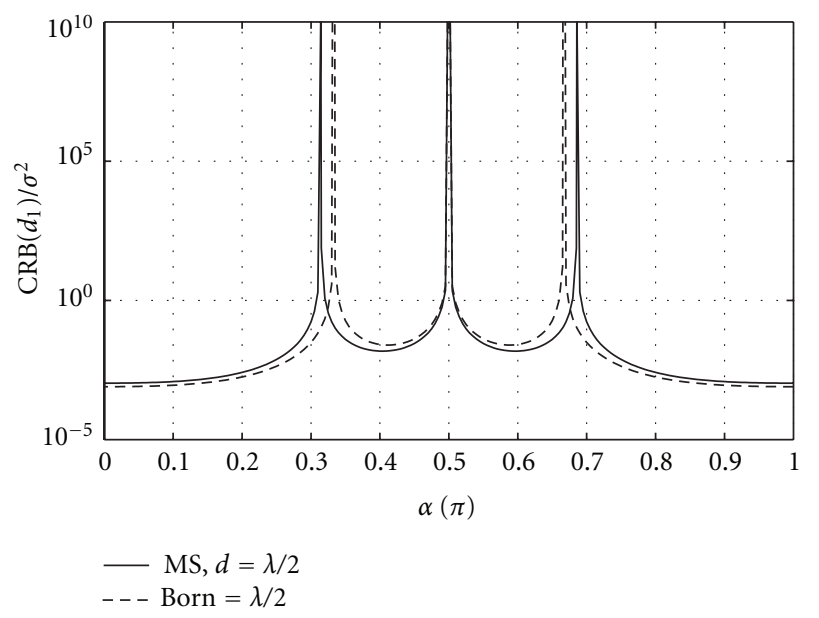

(b) $d=\lambda / 2$

Figure 5: Backscattering $\operatorname{CRB}\left(d_{1}\right)$ for $\tau_{1}=1=\tau_{2}$ versus the incidence angle $\alpha$ (where $\beta=\pi-\alpha$ ). Plots are provided for (a) $d=\lambda / 4$ and (b) $d=\lambda / 2$.

at approximately $\alpha=0.3143 \pi, 0.6857 \pi$ which closely match those of the Born model bound. Clearly the CRB behavior of both models is roughly equivalent. This recalls the point made in Section 4.2 that the nonlocalizable sets for the exact and approximate models are of equal size, which suggests (alongside this and other numerical examples to be shown next) that, overall, across the entire parameter space, the localizability predictions of the two models are rather comparable, a general conclusion of this study that is further highlighted next with more examples.

Figure 6 illustrates, for backscattering experiments and $\tau_{1}=1=\tau_{2}$, the $(d, \alpha)$-dependence of $\operatorname{CRB}\left(d_{1}\right)$ for $d \in$ $[0.1 \lambda, 10 \lambda]$ and $\alpha \in[0, \pi]$. The dark areas of the contour plot highlight regions where the bound peaks. The number of blind spots is seen to go up for higher values of $d$, in agreement with the discussion in (39) and (40). The exact results are essentially a smoothed-out version of the approximate results, with the general behavior of the two

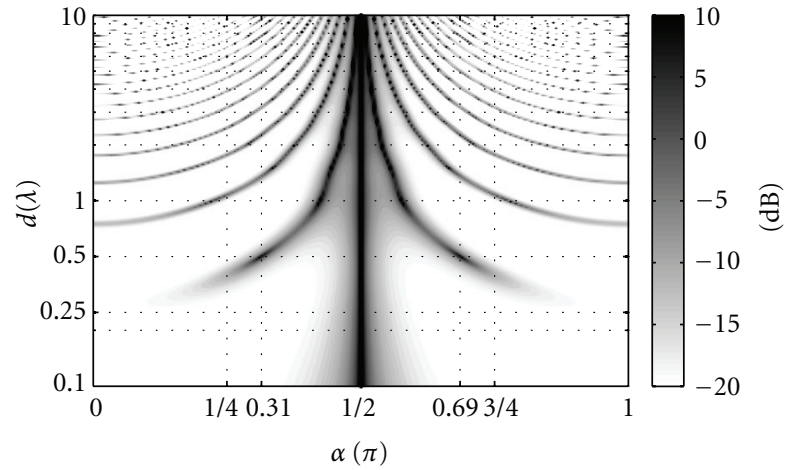

(a) Multiple scattering model

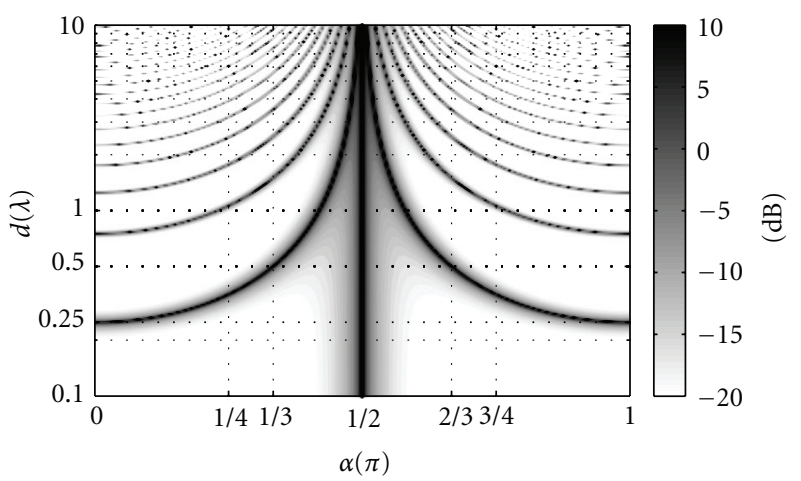

(b) Born approximation model

FIGURE 6: Contour plots of $\mathrm{CRB}\left(d_{1}\right)$ normalized by $\sigma^{2}$ corresponding to backscattering data for $\tau_{1}=1=\tau_{2}$ versus the incidence angle $\alpha$ and scatterers' separation $d$.

models being very similar for separation $d \gtrsim \lambda$. On the other hand, for separation $d \lesssim \lambda / 2$ the exact model does not exhibit the same blind spots as the Born approximation. Note that these results agree with the plots shown in Figure 5. Grid lines highlight the cross-sections of $d=\lambda / 4$ and $d=\lambda / 2$ corresponding to the results in Figure 5. For $d=\lambda / 2$, the grid line crosses 3 blinds at $\alpha=\pi / 3, \pi / 2$, and $2 \pi / 3$ for the Born approximation and $\alpha=0.3143 \pi, \pi / 2$, and $0.6857 \pi$ for the exact model, also highlighted with grid lines. Near $d=\lambda / 4$, the blinds asymptotically approach the horizontal $d=\lambda / 4$ boundary for the Born approximation but fade out before reaching it in the exact model, while the blind at $\alpha=\pi / 2$ is present in both cases since then $g=0$. Figure 7 illustrates further the relation between the Fisher information for the multiple scattering and Born approximation models. The regions, where $I\left(d_{1}\right) \leq I_{\text {Born }}\left(d_{1}\right)$ are shown in gray and black lines, show the blind conditions for the Born approximation model. Although not indicated explicitly in this figure, $I(d)=I_{\text {Born }}(d)$ along the line $\alpha=\pi / 2$ where both have zero Fisher information. This locus is the only one which bisects any of the regions where $I(d) \leq I_{\text {Born }}(d)$ in the figure. All others are only approached by these regions from one side. The checkered pattern for $d \gtrsim \lambda / 5$ indicates that the multiple scattering between the two targets alternates between aiding and impeding estimation of the target location as $\alpha$ and $d$ vary. The alternating pattern of 


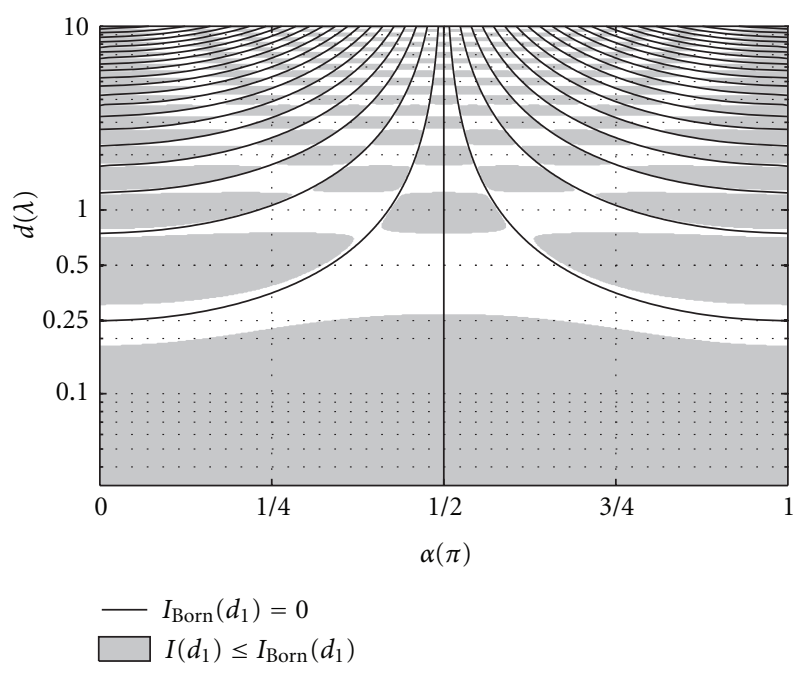

Figure 7: Map of the regions where multiple scattering effects do not aid estimation of target location in relation to blind conditions of the Born approximation model. Black lines mark conditions where $I_{\text {Born }}\left(d_{1}\right)=0$. Gray areas mark regions where $I\left(d_{1}\right) \leq$ $I_{\text {Born }}\left(d_{1}\right)$. The same parameters are used here as in Figure $6\left(\tau_{1}=\right.$ $\tau_{2}=1$, and $\left.\beta=\pi-\alpha\right)$.

helpful and pernicious multiple scattering effects in the exact model draws the ridges of high CRB in alternating directions adding a wavering quality to their shape. This can be seen in Figure 6(a). The same effect also breaks up the blind regions, so the multiple scattering does not produce the same continuous loci of infinite $\mathrm{CRB}\left(d_{1}\right)$ conditions as the Born approximation model. However, the bound remains very high in these areas so there may be little practical difference. For $d \lesssim \lambda / 5$, the curved blind condition loci produced by the nulls of the $\mathrm{SNR}_{\text {Born }}$ term in $I_{\text {Born }}\left(d_{1}\right)$ are no longer present and only the blind from the $g(\alpha, \beta)$ term at $\alpha=\pi / 2$ remains. Here the checkered pattern ends, and $I_{\text {Born }}\left(d_{1}\right)$ is consistently larger than $I\left(d_{1}\right)$ with the exception of the locus at $\alpha=\pi / 2$. This is consistent with the multiple observation example illustrated in a later section by Figure $8(\mathrm{a})$ where the error bounds of the multiple scattering and Born approximation models averaged over all observation and incidence angles converge above $d \approx \lambda / 2$ but for $d \lesssim \lambda / 5$ the CRB of the multiple scattering model is consistently higher. We conclude from these results that, in the particular backscattering setting, which is key for monostatic radar and sonar, the permissible target localization performance as measured by the fundamental CRB is in general comparable for the exact and approximate models, but that there are also clear differences between the two models as well as a number of well-defined regions (highlighted in the figures and discussion above), where multiple scattering clearly facilitates or obscures localizability.

5.2. Multiple Observations. So far we have emphasized the Fisher information and CRB predictions for estimating the target position with particular emphasis on single observations or SISO data. Our emphasis on this single observation

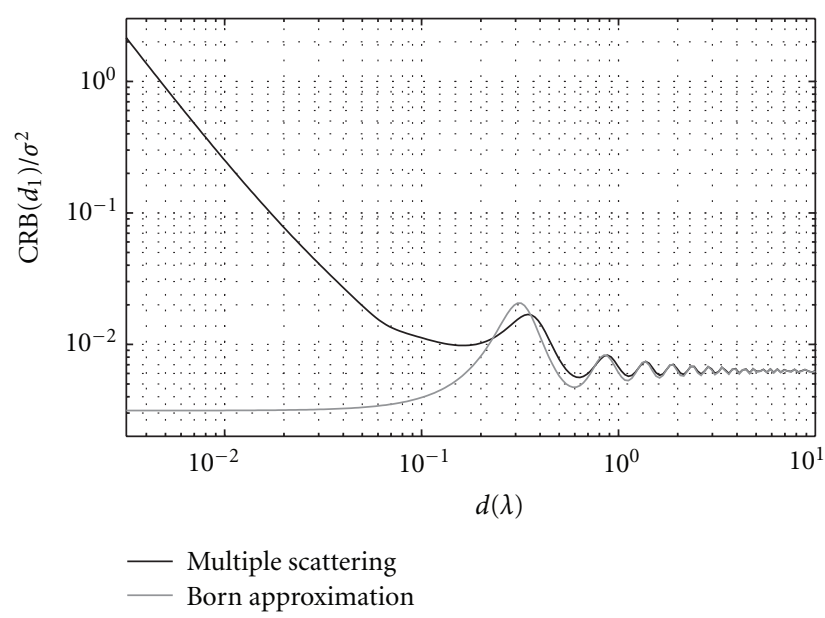

(a) $\tau_{1}=1, \tau_{2}=1$

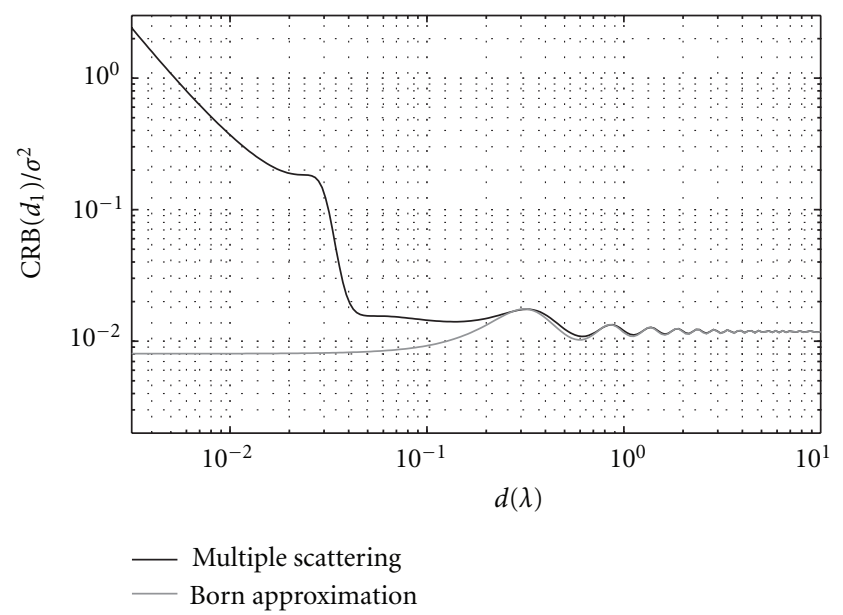

(b) $\tau_{1}=1, \tau_{2}=1 / 4$

Figure 8: Average $\operatorname{CRB}\left(d_{1}\right)$ as a function of the scatterers' separation $d$. The Fisher information on which the bound is based is averaged over $10^{4}$ data corresponding to 100 uniformly-spaced incidence and scattering angles in the full interval $[0, \pi]$. Two variations are shown: (a) equal $\left(\tau_{1}=1=\tau_{2}\right)$ and (b) unequal $\left(\tau_{1}=1\right.$ and $\left.\tau_{2}=1 / 4\right)$ scatterer strengths.

context has facilitated the extraction of analytically backed insight about the information content in the data under both the exact and the Born approximation models. A more general case is that in which multiple samples are available for each sensing configuration. The additional samples can increase SNR and therefore localizability. But in this case, the same general insight obtained above still applies. A completely different situation is that of multiple-input multiple-output (MIMO) data corresponding to different sensing configurations. We conclude by considering this case which is less transparent for analytical study. The MIMO calculations provide values of the CRB averaged over the sensing configuration parameters which effectively reduces the parameter dimensionality and allows us to decipher general patterns that are not obvious from the SISO analysis and associated computer results. 
Figure 8 shows plots of $\mathrm{CRB}\left(d_{1}\right)$ normalized by $\sigma^{2}$ versus $d$ for scattering data corresponding to MIMO experiments using 100 incidence angles $\alpha_{m}, m=1,2, \ldots, 100$ that are evenly spaced in the interval $[0, \pi]$, and identical scattering angles $\beta_{n}=\alpha_{n}, n=1,2, \ldots, 100$. The data are the $10^{4}$ entries of the resulting scattering matrix. Results are given for the two cases of (a) equally strength scatterers having $\tau_{1}=1=\tau_{2}$; (b) $\tau_{1}=1$ and $\tau_{2}=1 / 4$. The results for the two cases reveal the same general trend. In particular, we note that for $d \gtrsim \lambda / 2$ the predictions of the exact and approximate models are quite similar while, for $d \lesssim \lambda / 2$ the two models are very different, with the Born approximation CRB varying slowly and leveling off toward a plateau as $d$ becomes smaller, while the exact CRB increases drastically as $d$ becomes smaller. The value of $\operatorname{CRB}\left(d_{1}\right)$ shown is the average value of CRB per sample, as obtained by computing the average Fisher information and inverting it. From other experiments, we noted that good averages could be obtained with only $N_{t} \gtrsim 10$. The observed behavior is that the exact CRB is large for small target separation $d$ (below $0.1 \lambda$ ), but levels off and fluctuates slowly for $d>0.1 \lambda$. We found that for small $d$ the average $\operatorname{CRB}\left(d_{1}\right)$ is dominated by the term $1 /\left|F\left(d, \tau_{1}, \tau_{2}\right)\right|^{2}$, which is, according to (36) and (37), proportional to $\operatorname{CRB}\left(d_{1}\right)$ (see the plot of this term given in Figure 2). The term $F \rightarrow 0$ as $d \rightarrow 0$ so that from (37) the SNR goes to zero as $d \rightarrow 0$, and in turn from (36) also the localization information goes to zero as $d \rightarrow$ 0 . In other words, for small target separation the effect of multiple scattering is destructive in that it diminishes the SNR and consequently via (36) also the target localization information. This contrasts with the Born approximation calculation, where the information (and the respective CRB) tends in general to a finite value as $d \rightarrow 0$ as shown in the plots. In conclusion, these results suggest that, when averaged over all the sensing configurations, the exact CRB results are well-approximated by the Born approximation results for approximately $d \gtrsim \lambda / 2$. For approximately $\lambda / 10 \lesssim$ $d \lesssim \lambda / 2$, the two models differ visibly, and for $d \lesssim \lambda / \widetilde{10}_{0}$ the real $\mathrm{CRB}$ is significantly larger than the approximate $\mathrm{CRB}$, indicating that for such small separations the ability to estimate the position $d_{1}$ of the two-target system is highly reduced relative to what one would have expected from the Born approximation calculation. Physically, the reason is that then the multiple scattering produces destructive interference, reducing significantly the SNR of the received signal which is according to (36) the key quantity governing the target localizability.

\section{Conclusion}

This paper has explored the information about target position that is contained in scattering data by means of the fundamental statistical signal processing framework of the Fisher information and associated CRB pertinent to the unbiased estimation of scattering parameters. We have focused on the basic two-point scatterer system which is the simplest target exhibiting multiple scattering, with particular attention to the quantification of the target position information. To further facilitate formal tractability, we have emphasized the Fisher information and CRB calculations for all known scattering parameters (known two-point target case) except the target position. This approach has rendered closed-form expressions for the information and CRB for target position estimation, which has in turn offered a lot of mathematical and intuitive insight on the partly separate and partly combined roles of the sensing configuration and the target parameters and the companion internal multiple scattering. These developments have been elaborated further with the help of numerical illustrations.

The results have been derived in the exact multiple scattering framework as well as in the Born approximation model applicable only to weak scatterers exhibiting negligible multiple scattering. The multiple scattering results apply in general, and the Born approximation results are also important on their own since they apply to weak scatterers exhibiting negligible coupling between them. The commonalities and differences of the two models have been contrasted, and many concrete examples have been given of conditions under which multiple scattering has beneficial or detrimental effects in target localization relative to the single scattering framework. We have studied in detail the respective nonlocalizability conditions for both the exact multiple scattering model and the Born approximation model, concluding that while their NLOS blind spots generally differ, the sets of combined configuration and scattering parameters yielding nonlocalizability associated to these two models are actually equivalent. Thus in the entire configuration and scattering parameter space, the two models are comparable with regards to localizability. In addition, we have also discussed concrete necessary and sufficient conditions for the analog values of position information to be greater (or smaller) under multiple scattering versus the Born approximation. The results in this direction are important since they allow the quantification and understanding of the role of multiple scattering in localization without the need for explicitly evaluating the Fisher information and CRB. We have provided a detailed discussion of the blind spot conditions under which the target localization information is zero, for both scattering models, and specific examples of blind spots have been incorporated in the numerical illustrations section. The provided numerical results suggest that under broad-angle MIMO data, in which the effect of the particular sensing configuration is somehow averaged, the Born approximation model approximates well the exact multiple scattering results for values of $d \gtrsim \lambda / 2$, but that for $d \lesssim \lambda / 2$ the difference between the two is very marked. The MIMO results show that for small $d$ the effect of multiple scattering is consistently detrimental to localizability, thus for large scattering data sets and small separations $d$, the effect of multiple scattering appears to be destructive relative to the Born approximation whose associated predictions are therefore optimistic. The difference between the Born approximation and exact scattering models becomes visible also for specific single angle pairs or SISO configurations, where the role of the configuration can be decisively in favor (as enhancer of the target information) of one of the two models. On the other hand, as highlighted in 
the paper both theoretically and numerically, within the SISO framework the predictions of the two models regarding target localizability are in general comparable.

We plan to apply the techniques developed in this paper to other related problems such as estimation of the targets' separation which is related to the imaging resolution and quantification of the role of artificial or helper scatterers in target estimation and imaging. Finally, we wish to point out that clearly the results and conclusions derived in this study apply only insofar as the stated model assumptions are met. For instance, for simplicity we assumed an additive white noise model. Yet, multiplicative and correlated noise models are also relevant in practice. More importantly, the present developments hold only for monochromatic waves. Thus the questions addressed in the paper on the information content of scattering data with particular emphasis on the role of multiple scattering remain open for more general noise models and signals, for instance, broadband fields. These theoretical and practical issues provide interesting routes for future research.

\section{Acknowledgment}

This research was supported by the USA National Science Foundation under Grant 0746310.

\section{References}

[1] A. Sentenac, C. A. Guerin, P. C. Chaumet et al., "Influence of multiple scattering on the resolution of an imaging system: a Cramer-Rao analysis," Optics Express, vol. 15, no. 3, pp. 1340 1347, 2007.

[2] G. Shi and A. Nehorai, "Cramér-Rao bound analysis on multiple scattering in multistatic point-scatterer estimation," IEEE Transactions on Signal Processing, vol. 55, no. 6, pp. 2840-2850, 2007.

[3] F. Simonetti, M. Fleming, and E. A. Marengo, "Illustration of the role of multiple scattering in subwavelength imaging from far-field measurements," Journal of the Optical Society of America A, vol. 25, no. 2, pp. 292-303, 2008.

[4] X. Chen and Y. Zhong, "Influence of multiple scattering on subwavelength imaging: transverse electric case," Journal of the Optical Society of America A, vol. 27, no. 2, pp. 245-250, 2010.

[5] S. Kay, Fundamentals of Signal Processing: Estimation Theory, Prentice Hall, Wood Cliffs, NJ, USA, 1993.

[6] F. Simonetti, "Multiple scattering: the key to unravel the subwavelength world from the far-field pattern of a scattered wave," Physical Review E, vol. 73, no. 3, Article ID 036619, pp. $1-13,2006$.

[7] M. Shahram and P. Milanfar, "Imaging below the diffraction limit: a statistical analysis," IEEE Transactions on Image Processing, vol. 13, no. 5, pp. 677-689, 2004.

[8] M. Gustafsson and S. Nordebo, "Cramér-Rao lower bounds for inverse scattering problems of multilayer structures," Inverse Problems, vol. 22, no. 4, pp. 1359-1380, 2006.

[9] M. Zambrano-Nunez, E. A. Marengo, and D. Brady, "CramerRao study of scattering systems in one-dimensional space," in Proceedings of the International Conference on Antennas, Radar, and Wave Propagation (IASTED '10), November 2010.

[10] E. A. Marengo, M. Zambrano-Nuñez, and D. Brady, "CramerRao study of one-dimensional scattering systems: part I: formulation," in Proceedings of the 6th IASTED International Conference on Antennas, Radar, and Wave Propagation (ARP '09), pp. 1-8, July 2009.

[11] R. K. Snieder and J. A. Scales, "Time-reversed imaging as a diagnostic of wave and particle chaos," Physical Review E, vol. 58, no. 5, pp. 5668-5675, 1998.

[12] P. De Vries, D. V. Van Coevorden, and A. Lagendijk, "Point scatterers for classical waves," Reviews of Modern Physics, vol. 70, no. 2, pp. 447-466, 1998.

[13] M. Cheney and R. J. Bonneau, "Imaging that exploits multipath scattering from point scatterers," Inverse Problems, vol. 20, no. 5, pp. 1691-1711, 2004.

[14] L. Tsang, J. A. Kong, K.-H. Ding, and C. O. Ao, Scattering of Electromagnetic Waves: Numerical Simulations, John Wiley \& Sons, New York, NY, USA, 2001. 

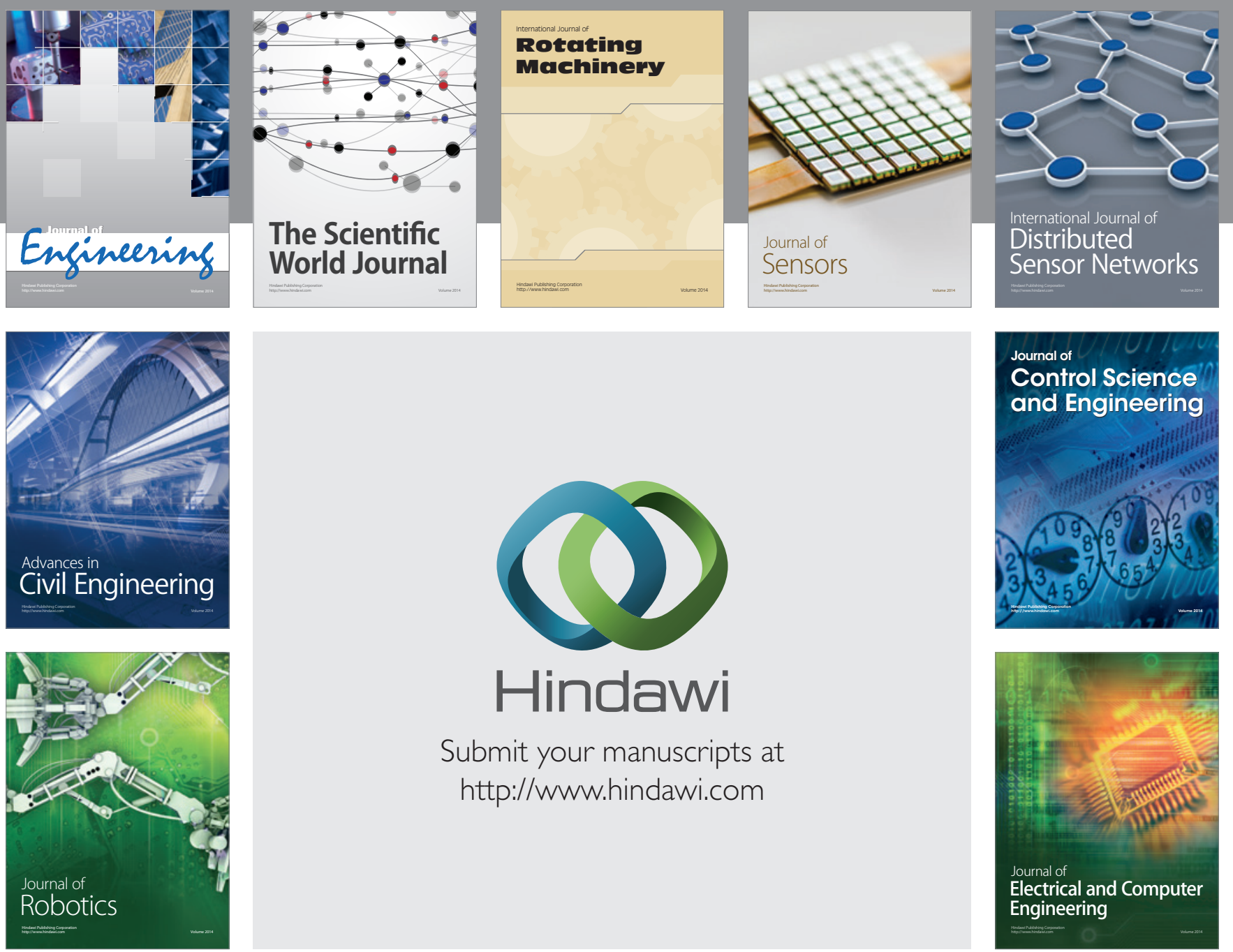

Submit your manuscripts at

http://www.hindawi.com
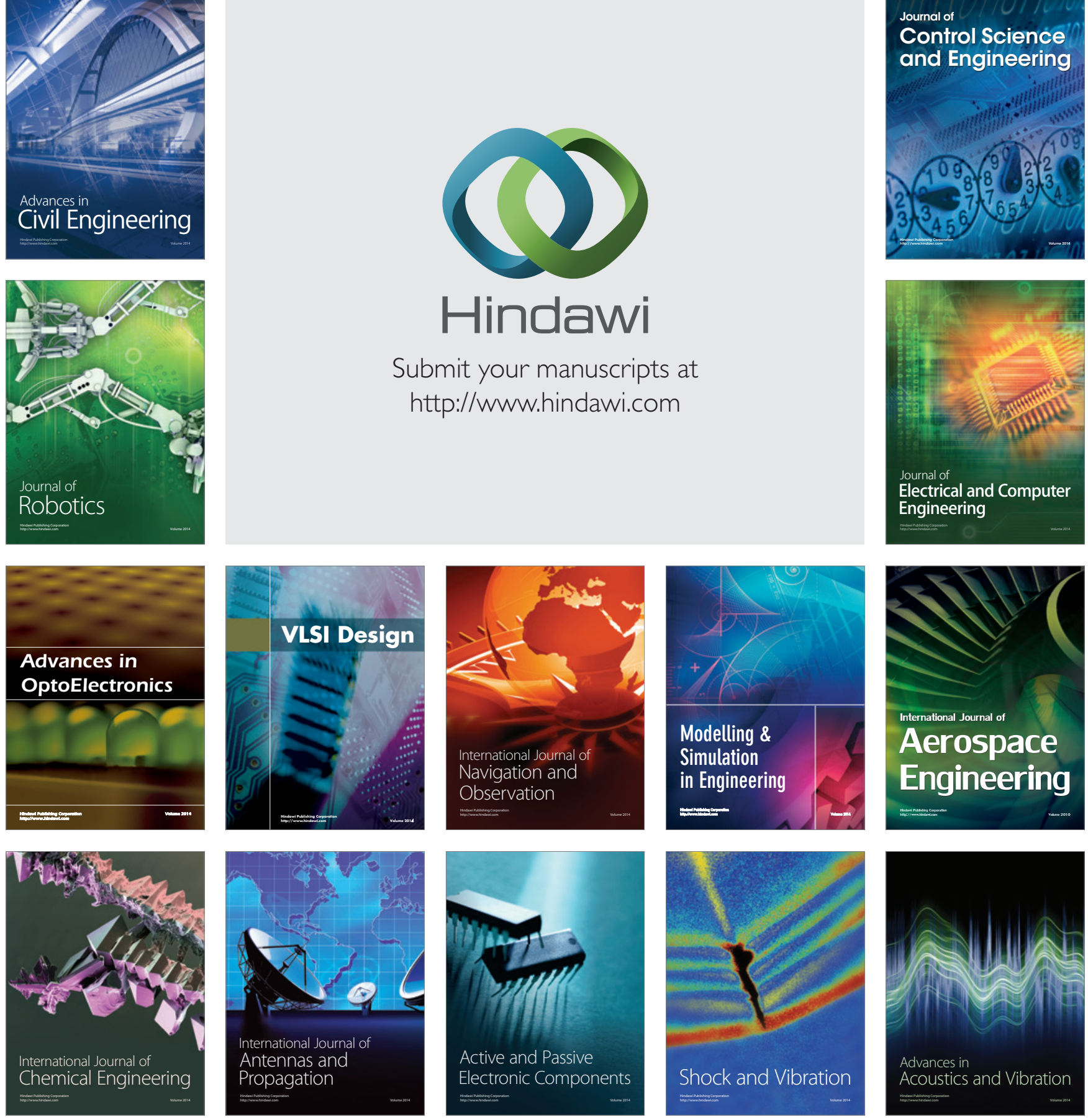